\title{
Hepatic biochemistry
}

Until the mid fifties the detailed study of intermediary metabolism in liver appeared to be remote from clinical medicine, and even from experimental pathology. This view was challenged by the observation that disease in visceral organs, notably liver and heart, was associated with striking changes in the activities of certain enzymes of intermediary metabolism in serum. Subsequently the specificity of these observations was increased by the demonstration that enzymes principally present in liver, such as glutamic pyruvic transaminase and glutamic dehydrogenase or, in muscle, such as creatine phosphokinase and aldolase, would show increased activity in serum as a consequence of injury to the parent organ. ${ }^{1}$ These observations have formed the basis of the increasing role of enzymology in diagnosis, and in monitoring the course of treatment. The extension of these findings to the identification of specific lesions in the parent tissue has, however, proved to be unrewarding. This is due to the fact that enzyme activity in tissues is usually many hundredfold or thousandfold greater than in serum. Marked changes in the activity of the enzyme in serum may therefore occur with only minimal loss of activity in the parent tissue, even if the loss of enzyme from tissue, or the disease process itself, did not serve as a stimulus for enzyme synthesis. Given such a high activity of enzyme in tissues, particularly liver, it became feasible to develop assay methods for use on small samples provided by percutaneous liver biopsies. The most extensive survey of a large number of enzymes of intermediary metabolism in diseases of the liver have been carried out by Schmidt and Schmidt ${ }^{2}$ resulting in the establishment of enzyme patterns characteristic of many acute and chronic forms of liver disease. An increase in the tissue of glucose-6-phosphate dehydrogenase, the enzyme initiating the pentose shunt pathway for the metabolism of glucose-6-phosphate, was found to be common to many forms of liver injury. Subsequently, Isselbacher and Jones ${ }^{3}$ demonstrated that in viral hepatitis of mice, and in experimental carbon tetrachloride poisoning, the amount of glucose-6-phosphate passing through the pentose shunt is indeed greater than in normal liver.

The liver is one of the few visceral organs readily accessible for biopsy, but fortunately many enzymes are quite stable at $25^{\circ} \mathrm{C}$. or below, as long as they remain in the tissue. The enzyme pattern of all the major human tissues could therefore be established, ${ }^{4}$ as well as that of tissues from experimental animals. In the latter, many key substrates of intermediary metabolism have likewise been measured. When these enzyme patterns and those of their associated substrates are examined they appear to be arranged in an almost random manner.

A more detailed study has, however, revealed that the relative activity of some groups of enzymes or of some enzymes with their substrates is similar in tissues as different as mammalian liver and insect flight muscle. These are referred to as 'constant proportion groups', and where located in the cytoplasm include the enzymes catalyzing the interconversion of the phosphorylated three-carbon compounds of glycolysis of the Emden-Meyerhof pathway between glyceraldehyde phosphate and phosphoenol pyruvate. ${ }^{5}$ In the mitochondria the malic dehydrogenase and glutamic oxalacetic transaminase located in these particles form a constant proportion group relative to cytochrome a with pyruvic oxidase and succinic dehydrogenase. ${ }^{6}$ Other mitochondrial enzymes, such as glycerol-1phosphate oxidase, isocitric dehydrogenase, and glutamic dehydrogenase, do not constitute a constant proportion group and their activities relative to one another are modified by the metabolic demands which different tissues are designed to meet.

A serious limitation of studies made on whole tissue homogenates is that when separating the major particulate components of the cell by differential centrifugation the need to homogenize the tissue remains. As a result differences which may exist at various sites within the liver lobule, perhaps due to differences in oxygen supply, are lost. Studies by Morrison, Shank, and their colleagues ${ }^{7}$ have demonstrated significant differences in enzyme activity in various parts of the liver lobule. These studies were based on assays of single cells or small numbers of isolated cells and the patience and perseverance required is given to few investigators. The more recent development of a gel diffusion 
method which appears to overcome many of the technical difficulties and possible artefacts of standard histochemical methods and which has confirmed the earlier results, is therefore especially welcome. ${ }^{8}$

A much more serious problem in the interpretation of enzyme assays is given by the fact that enzyme activity measured under optimal conditions in vitro and at high dilutions cannot be translated in vivo into the actual metabolic flow rates (i.e., the rate at which substrate is used up and product formed per unit weight of tissue, per unit time). Measurement of enzyme activity not only gives no direct information about the actual magnitude of flow, it does not even indicate the direction of metabolic flow in vivo. In general, enzyme activity measured in vitro represents no more than the maximal activity possible in vivo, and separate studies, such as those cited for glucose-6-phosphate dehydrogenase, are required to establish the significance of changes in enzyme activity. The calculation of actual, rather than maximal, metabolic flow rates in vivo is therefore difficult. Neglecting the possibilities of intracellular sequestration and compartmentation, the calculated flow rates must be determined by the enzyme activity, the relative concentrations of substrate(s) and product(s), their relationship to their concentration at equilibrium, as well as the mechanism of action of the enzyme under study. Activators and inhibitors can create situations of remarkable complexity. The best known example is the phosphofructokinase reaction, catalysing the transformation of fructose6-phosphate to fructose-1, 6,-diphosphate in the presence of adenosine triphosphatase. Depending to some extent on the tissue of origin and the concentration of the metabolites, the enzyme may be activated not only by its substrate, fructose-6-phosphate, but also by one of its products, fructose-1, 6-diphosphate, and the second product (A.D.P.) as well as by inorganic phosphate and A.M.P. The other substrate, A.T.P., acts as an inhibitor, the extent of the inhibition depending on the concentration of magnesium. The enzyme is also inhibited by phosphoenolpyruvate (an intermediate of glycolysis remote from the hexose phosphates) and by citrate. In liver the complexity of this step is compounded by the fact that an enzyme, fructose-1, 6-diphosphatase, having its own group of activators and inhibitors, catalyzes the reverse reaction: in other words, the formation of fructose-6-phosphate from fructose-1, 6-diphosphate. Meaningful calculations of flow rates through this step, in liver, in vivo therefore are not yet possible. If the substrate concentrations are near equilibrium, calculations of metabolic flow rates are perhaps less difficult. The most promising of the methods proposed is that of Bücher and Ruessman ${ }^{9}$ which is based on relatively simple and plausible assumptions, and capable of being applied without too much difficulty, at least to so-called twopartner reactions such as enolase and glucose-6-phosphate isomerase, i.e., reactions in which only one substrate and one product are involved.

It is fairly easy to separate in the laboratory mitochondria which are responsible for most of the oxygen taken up from the cytoplasmic fraction responsible for glycolysis. This may divert attention from the fact that these two cellular functions are related by the Pasteur and Crabtree effect, i.e., by the observation that respiration tends to inhibit glycolysis and that conversely, active glycolysis leads to a lowering of oxygen uptake. For oxidation to occur, hydrogen in the form of an oxidizable substrate must enter the mitochondria and eventually be oxidized by the electron transport chain, to combine with oxygen to form water. Some of the hydrogen is produced in the course of glycolysis as D.P.N.H. at the glyceraldehyde phosphate dehydrogenase step. The liver is the organ capable of taking up lactate produced in muscles and other tissues to form pyruvate and D.P.N.H. by means of the lactate dehydrogenase reaction; the D.P.N.H. level in the cytoplasm would therefore be expected to be high, especially in liver. In fact it is lower than in the mitochondria. This is all the more remarkable because D.P.N.H. cannot enter the mitochondria as such in vitro. For this reason a variety of cyclic shuttle systems have been postulated but most of these are either frankly hypothetical or cannot, on thermodynamic grounds, account for the apparently active accumulation of D.P.N.H. in the mitochondria. The only plausible system so far devised, the alpha-glycerophosphate cycle, can, in liver, account for at most $20 \%$ of the D.P.N.H. generated in the cytoplasm. ${ }^{10}$ The remaining $80 \%$ are of particular concern to the hepatologist because of the problem of disposing of the additional quantities of D.P.N.H. generated in the cytoplasm when alcohol is consumed and 
converted to D.P.N.H. and acetaldehyde by the alcohol dehydrogenase reaction.

In view of the recognized limitations of all studies in which the tissue is fragmented or destroyed, whole organ perfusion might be expected to overcome some of these difficulties. Until quite recently investigators have been reluctant to perfuse abnormal liver because in interpreting the data it is difficult to separate purely biochemical factors from those due to changes in the vascular anatomy and the reference base. It is therefore gratifying that data from two independent laboratories ${ }^{11,12}$ in which cirrhotic livers were perfused, using different techniques and different methods for the evaluation of results, agree in showing that oxygen consumption on a tissue weight basis is lowered in the perfused cirrhotic liver, and that contrary to some anticipations, uncontrolled 'dumping' of glucose from the cirrhotic liver does not occur. The lowered rate of consumption of oxygen is in agreement with earlier thoughts by Himsworth, ${ }^{13}$ but surprisingly is not reflected in the expected higher lactate to pyruvate ratios in the cirrhotic liver ${ }^{14}$ or in the perfusate. The relative normality of the lactate to pyruvate ratio (the classical indicator of tissue anoxia) in the cirrhotic is, however, readily disturbed when reducing equivalents are supplied in the form of ethanol and this disturbance appears to persist longer in the cirrhotic liver than in the normal. The oxygen supply of the cirrhotic liver is precarious at all times, and minimal disturbances in this state can result in striking and clinically important changes in tissue.

The approaches which have been outlined in this review-and some others which have not even been listed-are clearly complementary. None is without limitations, and each will provide relevant and interpretable information. Among the many studies which are currently being carried out in liver, the relationship between oxygen consumption, glucose utilization, and the quantitative identification of the intermediate steps which contribute to the observed differences in normal and cirrhotic liver is likely to occupy many laboratories fruitfully for many years to come.

KeITH S. HeNLEY

\section{REFERENCES}

${ }^{1}$ Henley, K. S., Schmidt, E., and Schmidt, F. W. (1966). Enzymes in Serum Their Use in Diagnosis. Thomas, Springfield, Ill.

${ }^{2}$ Schmidt, E., Schmidt, F. W., and Wildhirt, E. (1958-1963). (II-IV, VI-VIII, X, XII-XIII Ferment-Aktivitäets-Bestimmungen in der menschlichen Leber). Klin. Wschr. 36, 227-233, 280-287, 611-616 (1958). 37, 1221-1229, 1229-1230 (1959). 38, 421-428 (1960). 41, 637-643, 646-649, 649-653. (1963).

${ }^{3}$ Isselbacher, K. J., and Jones, W. A. (1964). Alterations of glucose metabolism in viral and toxic liver injury. Gastroenterology, 46, 424-433.

'Schmidt, E., Schmidt, F. W., Horn, H. U., and Gerlach, U. (1963). The importance of the measurement of enzyme activity in medicine. In Methods of Enzymatic Analysis, edited by H. U. Bergmeyer, pp. 651-712. Academic Press, New York.

${ }^{5}$ Pette, D., Luh, W., and Bücher, T., (1962). A constant-proportion group in the enzyme activity pattern of the Embden Meyerhof chain. Biochem, biophys. Res. Commun. 7, 419-424.

- Klingenberg, M., and Buecher, Th. (1962). Comparable and specific proportions in the mitochondrial enzyme activity pattern. Ibid., 7, 425-429.

'Morrison, G. R., Karl, I. E., Schwarts, R., and Shank, R. E. (1965). The quantitative histochemistry of the normal human liver lobule. J. Lab clin. Med., 65, 248-256.

8Pette, D., and Brandau, H. (1966). Enzym-Histiogramme und Enzymaktivitätsmuster der Rattenleber. Enzymol. biol. Clin., 6, 79-122.

'Bücher, Th., and Ruessman, W. (1964). Equilibrium and non-equilibrium in the glycolysis system. Angew. Chem. (int. Ed.)., 3, 426-439.

${ }^{10}$ Borst, P. (1963). Hydrogen transport and transport metabolites. In Funktionelle and Morphologische Organisation der Zelle, pp. $137-162$. Springer, Berlin.

"Salaspuro, M. P., and Maenpaa, P. H. (1966). Influence of ethanol on the metabolism of normal, fatty and cirrhotic livers. Biochem. J., 100, 768-774.

${ }^{12}$ Scholz, R., Grunst, J., Henley, K. S., Buecher, Th., and Hendelman, L. U. (1967). Metabolic studies with perfused cirrhotic liver. Gastroenterology, in the press.

${ }^{13}$ Himsworth, H. P. (1947). Lecture on the Liver and its Diseases. Harvard University Press.

"Henley, K. S., Gumucio, J. J., Laughrey, E. G., Hendelman, L. U., and Mueller, D. (1966). Glycolytic substrates in cirrhosis. Fed. Proc. 25,480 . 